\title{
INSTABILIDADE LINEAR DE CISALHAMENTO VERTICAL EM JATOS GEOFÍSICOS MERIDIONAIS
}

Ilson Carlos Almeida da Silveira', Leandro Calado², Cayo Prado Fernandes Francisco ${ }^{3}$

Submetido em 20 junho, 2003/Aceito em 29 abril, 2004

Submited June 20, 2003/Accepted April 29, 2004

\section{ABSTRACT}

The study of linear vertical shear instability of meridional (north-south) jets is conducted via an unidimensional hydrodynamic model, based on quasi-geostrophic potential vorticity conservation. The shape of the vertical jet profiles are motivated by the Brazil Current-Intermediate Western Boundary Current System that flows off the Brazilian Southeast coast and are built with the aim to satisfy necessary conditions for occuring baroclinic instability. The hydrodynamic model is based on Johns (1988) and its formulation is presented without the omission of algebraic details. Its physics is explored through numerically-solved examples and comparison with the analytical part of the model. The purpose is to subsidize the diagnosis of forms in which instability occur: at the ocean surface and in the interior of the water column. Additionally, the effect of the continental slope on the instability properties is investigated and discussed, through its effects on the propagation of unstable waves generated by instability process.

Keywords: geophysical jets, barodinic instability, Western Boundary Currents Brazil Current, unstable waves.

\section{RESUMO}

0 estudo da instabilidade linear de cisalhamento vertical (ou baroclínica) de perfis de jato de direção meridional (norte-sul) é conduzido através de um modelo hidrodinâmico unidimensional, baseado no princípio de conservação de vorticidade potencial quase-geostrófica. Os perfis verticais de jato utilizados nesse trabalho têm motivaçã̃o no Sistema Corrente do Brasil - Corrente de Contorno Intermediária que flui ao largo da costa sudeste brasileira. Os perfis estão construídos de forma a satisfazer as condições necessárias para ocorrência de instabilidade barodínica. 0 modelo hidrodinâmico é baseado no trabalho de Johns (1988) e tem sua formulação apresentada em detalhamento algébrico. Sua física é explorada através de exemplos e comparação com a parte anallíica do modelo, com o intuito de subsidiar o diagnóstico das formas com que a instabilidade ocorre na superfície do oceano e internamente na coluna de água. Adicionalmente, 0 efeito da inclinação do fundo associada ao talude continental é discutido e exemplificado, através de seus efeitos na propagaçãoo das ondas instáveis geradas pelos processos de instabilidade.

Palavras-chave. jatos geofísicos, instabilidade baroclínica, Corrente de Contorno Oeste, Corrente do Brasil, ondas instáveis.

Departamento de Oceanografia Física, Química e Geológica do Instituto Oceanográfico, Universidade de São Paulo, Praça do Oceanográfico, 191, 05508-900, Cidade Universitária, São Paulo SP - telefone: (11) 30916552 e-mail: ilson@io.usp.br

2 Departamento de Oceanografia Física, Química e Geológica do Instituto Oceanográfico, Universidade de São Paulo, Praça do Oceanográicico, 191, 05508-900, Cidade Universitária, São Paulo SP - telefone: (11) 30916578 e-mail: leandro@io.usp.br

3 Departamento de Oceanografia Física, Química e Geológica do Instituto Oceanográfico, Universidade de São Paulo, Praça do Oceanográfico, 191, 05508-900, Cidade Universitária, São Paulo SP - telefone: (11) 30916578 e-mail: cayoprado@io.usp.br 


\section{INTRODUÇÃO}

As correntes de contorno oeste são jatos geofísicos caracterizados como fluxos intensos, estreitos e bem definidos, fluindo ao largo das margens continentais oeste das bacias oceânicas. A Corrente do Brasil (CB) é uma dessas correntes de contorno que flui bordejando a costa Sudeste do Brasil e em seu percurso apresenta a formação de largos meandros, que ocasional mente se fecham em vórtices. A formação dessas estruturas tem sido bastante descrita na literatura (SILVEIRA et al., 2001; EVVANS; SIGNORINI, 1985).

Resultados recentes de modelagem numérica (CALADO, 2001; FERNANDES, 2001) mostram que 0 crescimento dos meandros se deve a drenagem de energia potencial do escoamento médio da CB. Estes meandros, que nascem com pequena amplitude, crescem exponencialmente. Efeitos não-lineares se tornam importantes $\mathrm{e}$ eventualmente ocorre 0 isolamento do meandro e 0 destacamento de vórtices do eixo do jato.

Tal processo de crescimento de meandros sugere a ocorrência de um fenômeno de instabilidade hidrodinâmica, que pode ser explicado por teoria linear. A instabilidade hidrodinâmica, em questão, é a instabilidade de cisalhamento vertical ou instabilidade baroclínica.

A estrutura vertical de velocidade da coluna de água da região na CB é caracterizada por singular variação em termos de oceano mundial. Até os primeiros $300-400 \mathrm{~m}$ de profundidade a CB flui em direção a sulsudeste. A partir deste limite de profundidade, há uma inversão de fluxo para norte-nordeste, associado a uma outra corrente, denominada Corrente de Contorno Oeste Intermediária (CCI). Esta tem seu núcleo máximo de velocidade, centrado em aproximadamente $800-1000 \mathrm{~m}$ de profundidade (SILVEIRA et al., 2001).

Motivado pelo cisalhamento vertical singular do sistema CB-CCl, construiu-se um modelo linear unidimensional de instabilidade barodínica para um jato meridional (de direção norte-sul) que inverte 0 sentido ao longo da coluna de água num oceano continuamente estratificado. Este modelo é baseado no trabalho de Johns (1988) e usa os preceitos dinâmicos de Gill e outros (1974).

0 objetivo central deste trabalho é a avaliação das condições necessárias para ocorrência de instabilidade baroclínica e descrição do diagnóstico da forma com que esta instabilidade ocorre. Adicionalmente, detalhamento da derivação do modelo será fornecida.

\section{MODELO DE INSTABILIDADE BAROCLÍNICA}

\section{Formulação do modelo}

Considerando uma porção da superfície da Terra aproximada por um plano girando com velocidade angular constante $f / 2,0$ chamado plano " $f$ ", construiu-se um modelo para o estudo das propriedades de instabilidades de cisalhamento vertical em um escoamento fluído de orientação norte-sul.

0 modelo parte da equação de conservação da vorticidade potencial, que representa um princípio análogo ao da conservação de momento angular da mecânica clássica. Assim,

$\frac{\mathrm{dq}}{\mathrm{dt}}=\frac{\partial \mathrm{q}}{\partial \mathrm{t}}+\frac{\partial \mathrm{q}}{\partial \mathrm{x}}+\frac{\partial \mathrm{q}}{\partial \mathrm{y}}=0$

onde q é a vorticidade potencial (VP) quase-geostrófica, $v$ é velocidade meridional (norte - sul) e u a velocidade zonal (leste - oeste).

Assumindo um escoamento horizontalmente não-divergente, define-se a função $\psi$ denominada função de corrente geostrófica por

$\vec{V}=u \overrightarrow{1}+v \vec{j}=\vec{k} \times \nabla \Psi$,

e reescreve-se a Equação (1) como

$\frac{\mathrm{dq}}{\mathrm{dt}}=\frac{\partial \mathrm{q}}{\partial \mathrm{t}}+\mathrm{J}(\Psi, \mathrm{q})=0$.

Utilizando-se o método das perturbações, define-se quantidades médias e perturbadas para as seguintes variáveis:

$\mathrm{q}=\overline{\mathrm{q}}(\mathrm{x}, \mathrm{y}, \mathrm{z})+\mathrm{q}^{\prime}(\mathrm{x}, \mathrm{y}, \mathrm{z}, \mathrm{t})$,

$\Psi=\bar{\Psi}(\mathrm{x}, \mathrm{y}, \mathrm{z})+\mathrm{q}^{\prime}(\mathrm{x}, \mathrm{y}, \mathrm{z}, \mathrm{t})$,

$\mathrm{u}=\mathrm{u}^{\prime}(\mathrm{x}, \mathrm{y}, \mathrm{z}, \mathrm{t})$,

$\mathrm{v}=\overline{\mathrm{v}}(\mathrm{x}, \mathrm{y}, \mathrm{z})+\mathrm{v}^{\prime}(\mathrm{x}, \mathrm{y}, \mathrm{z}, \mathrm{t})$ 
as quantidades $\overline{\mathrm{q}}, \bar{\Psi}$ e $\overline{\mathrm{v}}$ são valores médios e q', $\Psi$, e u' representam as quantidades perturbadas. Os campos médios são construídos de forma a serem paramétricos em y o que implica em considerarmos os campos básicos são invariantes por translação meridional.

Substituindo-se as igualdades acima em (3), chega-se a:

$$
\begin{aligned}
& \frac{d q}{d t}=\frac{\partial q^{\prime}}{\partial t}+u^{\prime}\left[\frac{\partial \bar{q}}{\partial x}+v \frac{\partial q^{\prime}}{\partial x}\right]+ \\
& +\bar{v}\left[\frac{\partial \bar{q}}{\partial y}+v \frac{\partial q^{\prime}}{\partial y}\right]+v^{\prime}\left[\frac{\partial \bar{q}}{\partial Y}+v \frac{\partial q^{\prime}}{\partial y}\right]=0
\end{aligned}
$$

Linearizando-se a Equação (8) e admitindo-se que $\bar{q}$ seja uma função paramétrica de $y$, obtém-se :

$$
\left[\frac{\partial}{\partial t}+\bar{v} \frac{\partial}{\partial y}\right] q^{\prime}+u^{\prime} \frac{\partial \bar{q}}{\partial x}=0
$$

tal que $\overline{\mathrm{q}}$ e q', de acordo com Pedlosky (1979) e Cushman-Roisin (1994), são dados por

$$
\begin{aligned}
& \bar{q}=\frac{\partial}{\partial z} \frac{f^{2}}{N^{2}} \frac{\partial \bar{\Psi}}{\partial z}+\frac{\partial^{2} \Psi}{\partial x^{2}}, e \\
& q^{\prime}=\frac{\partial}{\partial z} \frac{f^{2}}{N^{2}} \frac{\partial \Psi}{\partial z}+\nabla^{2} \Psi,
\end{aligned}
$$

onde $f$ é 0 valor do parâmetro de Coriolis médio na região, $\bar{v}$ é o perfil vertical médio de velocidade meridional e $\mathrm{N}^{2}$ é a frequência de BruntVäisällä ou frequência de empuxo.

0 primeiro termo das relações anteriores (equaçōes 10 e 11) representa a contribuição à vorticidade do estiramento ou achatamento de uma parcela de fluido que é refletida na divergência (achatamento) ou convergência (estiramento) horizontais do fluido. 0 segundo termo, em ambas as relações, representa a vorticidade relativa, que é a vorticidade devida ao cisalhamento horizontal das velocidades.

A frequência de empuxo, por sua vez, representa uma medida da variação vertical da densidade $\rho$ do meio dada por:

$$
\mathrm{N}^{2}(\mathrm{z})=\frac{-\mathrm{g}}{\rho_{0}} \frac{\partial \rho}{\partial \mathrm{z}}
$$

onde $\rho_{0}$ é a densidade média da coluna de água e $\mathrm{g}$ a aceleração da gravidade.
Para o propósito de aplicações futuras, pode-se, através de uma análise de escala, simplificar a Equação (10) verificando que $\frac{\partial^{2} \bar{\Psi}}{\partial \mathrm{x}^{2}} \ll \frac{\partial}{\partial z} \frac{\mathrm{f}^{2}}{\mathrm{~N}^{2}} \frac{\partial \bar{\Psi}}{\partial z}$.Dessa forma a Equaçã̃o (9) é reescrita na forma: $\left(\frac{\partial}{\partial t}+\bar{v} \frac{\partial}{\partial y}\right)\left(\nabla^{2} \Psi^{\prime}+\frac{\partial}{\partial z} \frac{f^{2}}{N^{2}} \frac{\partial \Psi^{\prime}}{\partial z}\right)+u \cdot\left(\frac{\partial}{\partial z} \frac{f^{2}}{N^{2}} \frac{\partial \bar{v}}{\partial z}\right)=0$

As condições de contorno apropriadas para aplicações oceanográficas exigem que se tenha velocidade vertical nula nos limites superior e inferior do oceano, isto é $\mathrm{w}=0$ em $\mathrm{z}=0, \mathrm{H}$. Para superfície $(\mathrm{z}=\mathrm{H})$, de acordo com Flierl (1978), pode-se, através de argumentos termodinâmicos, considerar que:

$$
\mathrm{w}=\frac{\mathrm{d} \eta}{\mathrm{dt}},
$$

onde $\eta$ representa 0 desvio de uma superfície de densidade constante relativamente a seu nível de equilibrio hidrostático. Utilizando-se a equação hidrostática, tem-se $\eta$ em função de $\psi$

$\eta=-\frac{\mathrm{f}}{\mathrm{N}^{2}} \frac{\partial \Psi}{\partial \mathrm{z}}$

Combinando (15) com (14) e utilizando-se a condição $\mathrm{w}=0$ chegamos a :

$$
\frac{\mathrm{d} \frac{\partial \Psi}{\partial \mathrm{z}}}{\mathrm{dt}}=0 \text {. }
$$

Utilizando-se a definição de $\psi$ (Equação 5) e desprezando-se os termos não-lineares obtém-se

$$
\left(\frac{\partial}{\partial \mathrm{t}}+\overline{\mathrm{v}} \frac{\partial}{\partial \mathrm{y}}\right) \frac{\partial \Psi}{\partial \mathrm{z}}=\frac{\partial \overline{\mathrm{v}}}{\partial \mathrm{z}} \frac{\partial \Psi}{\partial \mathrm{y}} \text { para } \mathrm{z}=\mathrm{H} .
$$

Para o fundo, assumimos uma pequena variação topogrática de orientação zonal $\mathrm{h}=\mathrm{h}(\mathrm{x})$,

$$
\mathrm{w}=\frac{\mathrm{dh}}{\mathrm{dt}}=0 \text {. }
$$

Linearizando-se a condição de contorno de fundo, analogamente à da superfície, chega-se a:

$\left(\frac{\partial}{\partial \mathrm{t}}+\overline{\mathrm{v}} \frac{\partial}{\partial \mathrm{y}}\right) \frac{\partial \Psi}{\partial \mathrm{z}}-\frac{\partial \overline{\mathrm{v}}}{\partial \mathrm{z}} \frac{\partial \Psi}{\partial \mathrm{y}}=\frac{\partial \Psi}{\partial \mathrm{y}} \frac{\partial \mathrm{h}}{\partial \mathrm{x}} \frac{\mathrm{N}^{2}}{\mathrm{f}^{2}}$,

ou rearranjando-se os termos,

$$
\left(\frac{\partial}{\partial \mathrm{t}}+\overline{\mathrm{v}} \frac{\partial}{\partial \mathrm{y}}\right) \frac{\partial \Psi^{\prime}}{\partial \mathrm{z}}=\frac{\partial \overline{\mathrm{v}}}{\partial \mathrm{z}}\left(1+\frac{\partial \mathrm{h}^{*}}{\partial \mathrm{x}}\right) \frac{\partial \Psi}{\partial \mathrm{y}} \operatorname{para} z=0,
$$


onde $\frac{\partial \mathrm{h}^{*}}{\partial \mathrm{x}}=\mathrm{N}^{2} \frac{\partial \mathrm{h}}{\partial \mathrm{x}} / \mathrm{f}^{2} \frac{\partial \overline{\mathrm{v}}}{\partial \mathrm{z}}$, é a razão entre a inclinação do assoalho oceânico e a inclinação das linhas de mesma densidade (isopicnais) junto ao fundo.

Conforme descrito na Seção 1, objetiva-se isolar o efeito da instabilidade de cisalhamento vertical, em que as flutuações drenam energia primariamente do campo médio de energia potencial. Para isto deve-se considerar os campos $\bar{v}, \mathrm{~N}^{2}$ e,

$$
\frac{\partial \bar{q}}{\partial x}=\frac{\partial}{\partial z} \frac{f^{2}}{N^{2}} \frac{\partial \bar{v}}{\partial z},
$$

independentes de $\mathrm{x}$, ou seja, elimina-se 0 efeito do cisalhamento horizontal e unidimensionaliza-se o problema.

Deve-se também impor a condição de contorno lateral a fim de fechar o problema de instabilidade. Tais condições de contorno são impostas de forma a confinar as perturbações em uma região definida como um canal de largura $2 \mathrm{~L}$, que remete à idéia de um jato meridional limitado lateralmente em $\mathrm{x}= \pm \mathrm{L}$ (Figura 1). Portanto, as condições de contorno laterais implicam que não há escoamento normal aos limites laterais do canal. Assim,

$$
\frac{\partial \Psi}{\partial \mathrm{Y}}=0 \mathrm{em} \mathrm{x}= \pm \mathrm{L}
$$

onde L é a largura do canal.

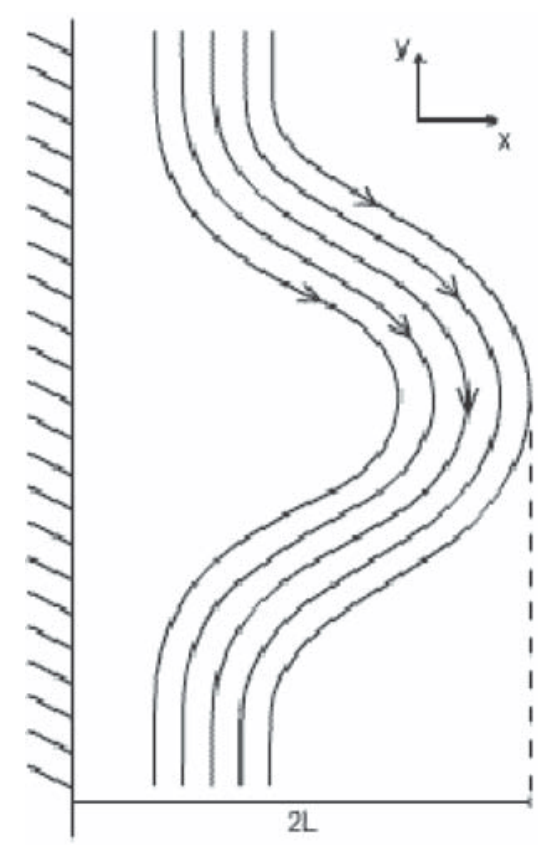

Figura 1 - Esquema no plano xy da perturbação zonal no jato meridional. Figure 7 - Scheme on the xy - plane of the zonal disturbance of the meridional jet.
Para a resolução do sistema de equações formado por (11), (17) e (20), escolhe-se soluções ondulatórias que sejam periódicas em y e confinadas na região $\mathrm{x}= \pm \mathrm{L}$ da forma

$$
\Psi^{\prime}(\mathrm{x}, \mathrm{y}, \mathrm{z}, \mathrm{t})=\Psi(\mathrm{z}) \cos \left(\frac{\pi \mathrm{x}}{2 \mathrm{~L}}\right) \mathrm{e}^{-\mathrm{ik}(\mathrm{y}-\mathrm{ct})} .
$$

tal que $\Psi$ (z) é a amplitude da perturbação e c é a e velocidade de fase das ondas. A solução considera perturbações em $\mathrm{x}$, substituindo (23) na (19)

$$
\begin{aligned}
& \left(\frac{\partial}{\partial \mathrm{t}}+\overline{\mathrm{v}} \frac{\partial}{\partial \mathrm{y}}\right)\left[-\Psi\left(\frac{\pi}{2 \mathrm{~L}}\right)^{2}-\mathrm{k}^{2} \Psi+\frac{\partial}{\partial \mathrm{z}} \frac{\mathrm{f}^{2}}{\mathrm{~N}^{2}} \frac{\partial \Psi}{\partial \mathrm{z}}\right]+ \\
& +\mathrm{ik} \Psi \frac{\partial \overline{\mathrm{q}}}{\partial \mathrm{x}}=0
\end{aligned}
$$

$$
\begin{aligned}
& {\left[\left(\frac{\pi}{2 \mathrm{~L}}\right)^{2}(\mathrm{c}-\overline{\mathrm{v}})+\mathrm{k}^{2}(\mathrm{c}-\overline{\mathrm{v}})+(\mathrm{c}-\overline{\mathrm{v}}) \frac{\partial}{\partial \mathrm{z}} \frac{\mathrm{f}^{2}}{\mathrm{~N}^{2}} \frac{\partial}{\partial \mathrm{z}}\right]} \\
& \Psi-\Psi \frac{\partial \overline{\mathrm{q}}}{\partial \mathrm{x}}=0
\end{aligned}
$$

dividindo-se Equação (25) por ( $\mathrm{c}-\overline{\mathrm{v}})$, tem-se,

$$
\frac{\partial}{\partial \mathrm{z}}\left(\frac{\mathrm{f}^{2}}{\mathrm{~N}^{2}} \frac{\partial \Psi}{\partial \mathrm{z}}\right)-\left[\mathrm{k}^{2}+\frac{\pi^{2}}{4 \mathrm{~L}^{2}}+\frac{1}{(\overline{\mathrm{v}}-\mathrm{c})} \frac{\partial \overline{\mathrm{q}}}{\partial \mathrm{x}}\right] \Psi=0 \text {. }
$$

Com um procedimento similar, substituindo a Equação (23) nas condições de contorno (Equações (17) e (20)),

$$
\begin{aligned}
& (\overline{\mathrm{v}}-\mathrm{c}) \frac{\partial \Psi}{\partial \mathrm{z}}=\Psi \frac{\partial \overline{\mathrm{v}}}{\partial \mathrm{z}} \text { em } \mathrm{z}=\mathrm{H} \\
& (\overline{\mathrm{v}}-\mathrm{c}) \frac{\partial \Psi}{\partial \mathrm{z}}=\left(1-\frac{\partial \mathrm{h}^{*}}{\partial \mathrm{x}}\right) \Psi \frac{\partial \overline{\mathrm{v}}}{\partial \mathrm{z}} \text { em } \mathrm{z}=0
\end{aligned}
$$

Utilizando-se perfis médios de $\bar{v}$ e $\mathrm{N}$, resolvendo 0 conjunto de Equações (26), (27) e (28) numericamente, como um problema de autovalores, obtém-se as amplitudes e velocidades de fase das ondas.

\section{ANÁLISE DE INSTABILIDADE}

A equação para análise das condições necessárias, mas não suficientes para a ocorrência de instabilidade baroclínica, pode ser obtida pela multiplicação da Equação (26) pelo complexo conjugado $\Psi^{*}$ da função de corrente, sendo : 
$\left(\frac{\partial}{\partial \mathrm{z}} \frac{\mathrm{f}^{2}}{\mathrm{~N}^{2}} \frac{\partial \Psi}{\partial \mathrm{z}}\right) \Psi^{*}-\left[\mathrm{k}^{2}+\frac{\pi^{2}}{4 \mathrm{~L}^{2}}+\frac{1}{(\overline{\mathrm{v}}-\mathrm{c})} \frac{\partial \overline{\mathrm{q}}}{\partial \mathrm{x}}\right] \Psi^{2}=0$.

Integrando a Equação (29) da superfície ao fundo e retendo apenas os termos complexos, obtém-se,

$\underbrace{\int_{0}^{\mathrm{H}}\left(\frac{\partial}{\partial \mathrm{z}} \frac{\mathrm{f}^{2}}{\mathrm{~N}^{2}} \frac{\partial \Psi}{\partial \mathrm{z}}\right) \Psi^{*} \mathrm{dz}}_{\mathrm{a}}-\underbrace{\int_{0}^{\mathrm{H}} \frac{1}{(\bar{\nu}-\mathrm{c})} \frac{\mathrm{d} \overline{\mathrm{q}}}{\partial \mathrm{x}} \Psi^{2} \mathrm{dz}}_{\mathrm{b}}=0$,

integrando por partes 0 primeiro termo (a) :

$\int_{0}^{\mathrm{H}}\left(\frac{\partial}{\partial \mathrm{z}} \frac{\mathrm{f}^{2}}{\mathrm{~N}^{2}} \frac{\partial \Psi}{\partial \mathrm{z}}\right) \Psi^{*} \mathrm{dz}=\left.\frac{\mathrm{f}^{2}}{\mathrm{~N}^{2}} \frac{\partial \Psi}{\partial \mathrm{z}} \Psi^{*}\right|_{0} ^{\mathrm{H}}-\int_{0}^{\mathrm{H}} \frac{\mathrm{f}^{2}}{\mathrm{~N}^{2}} \frac{\partial \Psi}{\partial \mathrm{z}} \frac{\partial \Psi^{*}}{\partial \mathrm{z}} \mathrm{dz}$,

Pode-se perceber facilmente que 0 termo sob a operação de integraçacoo, à direita da igualdade, é real e portanto iremos preservar apenas 0 primeiro termo, este será avaliado nos seus extremos utilizandose as condições de contorno - equaç̃oes (27) e (28)). Assim,

$\left.\frac{\partial \Psi}{\mathrm{dz}} \Psi^{*}\right|_{0} ^{\mathrm{H}}=\frac{\mathrm{f}^{2}}{\mathrm{~N}^{2}} \frac{\frac{\partial \bar{\nu}}{\partial \mathrm{z}} \Psi \Psi^{*}}{\bar{\nu}-\mathrm{c}}-\frac{\mathrm{f}^{2}}{\mathrm{~N}^{2}} \frac{\frac{\partial \bar{v}}{\partial \mathrm{z}}\left(1-\mathrm{h}_{\mathrm{x}}^{*}\right)}{\bar{\nu}-\mathrm{c}} \Psi \Psi^{*}$,

a expressão obtida para os extremos pode então ser reescrita na forma

$\left(\underset{\text { real }}{\bar{\nu}}+\underset{c_{r}+i_{i}}{c}\right) \frac{f^{2}}{N^{2}}\left[\frac{\frac{\partial \bar{v}}{\partial} \Psi^{2}}{|\bar{\nu}-c|^{2}}-\frac{\frac{\partial \bar{v}}{\partial z}\left(1-h_{x}^{*}\right) \Psi^{2}}{|\bar{v}-c|^{2}}\right]$.

0 segundo membro (b), por sua vez, pode ser reescrito como

$(\bar{\nu}+\mathrm{c}) \int_{0}^{\mathrm{H}} \frac{\frac{\partial \overline{\mathrm{q}}}{\partial \mathrm{x}}}{|\bar{v}-\mathrm{c}|^{2}} \Psi^{2} \mathrm{dz}$.

Considerando-se, então, somente a parte imaginária das integrações dos termos (a) e (b), obtemos

$\mathrm{c}_{\mathrm{i}}\left[\mathrm{f}^{2}\left[\left(\left[\frac{\frac{\partial \bar{\nu}}{\partial \mathrm{z}} \Psi^{2}}{\mathrm{~N}^{2}|\bar{\nu}-\mathrm{c}|^{2}}\right]_{\mathrm{H}}-\left[\frac{\frac{\partial \bar{\nu}}{\partial \mathrm{z}}\left(1-\mathrm{h}_{\mathrm{x}}^{*}\right) \Psi^{2}}{\mathrm{~N}^{2}|\bar{\nu}-\mathrm{c}|^{2}}\right]_{0}\right)-\int_{0}^{\mathrm{H}} \frac{\frac{\partial \overline{\mathrm{q}}}{\mathrm{x}}}{|\bar{\nu}-\mathrm{c}|^{2}} \Psi^{2} \mathrm{dz}\right]=0\right.$.

\section{CONDIÇÕES NECESSÁRIAS PARA INSTABILIDADE}

De acordo com a Equação (35), pode-se dizer que, para as ondas instáveis, a condição $\mathrm{c}_{\mathrm{i}}>0$ tem de ser satisfeita. Para tanto, os termos entre colchetes devem se anular. As condições necessárias, mas não suficientes para que isto ocorra, são:

- $\frac{\partial \overline{\mathrm{q}}}{\partial \mathrm{x}}$ trocar de sinal entre a superfície e o fundo; ou - se em alguma profundidade, $\frac{\partial \bar{q}}{\partial x}$ tiver o mesmo sinal que $\frac{\partial \bar{v}}{\partial z}$ na
superfície; ou

- se em alguma profundidade, $\frac{\partial \overline{\mathrm{q}}}{\partial \mathrm{x}}$ tiver sinal oposto à quantidade $\left(1-\frac{\partial h^{*}}{\partial x}\right) \frac{\partial \bar{v}}{\partial z}$ junto ao fundo.

Logo, a partir do perfil do gradiente de vorticidade potencial, pode-se inferir, à luz da teoria de estabilidade linear, se 0 jato é "potencialmente" baroclinicamente instável ou não. 0 problema da inferência da instabilidade baroclínica de um jato meridional fica dependente especificamente dos perfis de $\bar{v}, \mathrm{~N}$ e $\frac{\partial \overline{\mathrm{q}}}{\partial \mathrm{x}}$.

Um jato instável pode gerar ondas de períodos e velocidades de fase distintas. Consegue-se diagnosticar diferentes tipos de instabilidade baroclínica, analisando-se o período e a velocidade de fase das ondas instáveis.

As instabilidades abordadas neste trabalho são, basicamente, duas. A primeira, instabilidade de borda (EADY, 1949; GREEN, 1960), a segunda, instabilidade interna (CHARNEY, 1947; CHARNEY; STERN, 1962).

Estes dois tipos de instabilidades podem ser diagnosticadas comparando-se o nível de acoplamento entre as velocidades de fase das ondas instáveis e as velocidades do perfil de corrente. 0 nível de acoplamento é definido como o nível no qual $\bar{v}=c_{r}$ (PEDLOSKY, 1979), ou seja: onde as ondas instáveis se "acoplam" ao fluxo médio para dele drenar energia potencial.

A instabilidade de borda pode ser caracterizada por ondas instáveis que tenham nível de acoplamento das velocidades de fase próximo ao das velocidades nos limites superiores ou inferiores do perfil de velocidade, isto é $\bar{v}=c_{r}$ nos contornos. Se o nível de acoplamento estiver em uma região próxima a um dos contornos, as ondas instáveis serão aprisionadas próximas a este.

Já para a instabilidade interna, o nível de acoplamento se dá com valores das velocidades de fase das ondas instáveis próximos aos valores de velocidades do interior do campo, isto é $\bar{v}=c_{r}$ no interior do jato e, por conseqüência, da coluna de água. É característico deste tipo de instabilidade gerar ondas que tenham velocidades de fase com valores nas vizinhanças da interface da inversão de sinal no campo de $\frac{\partial \overline{\mathrm{q}}}{\partial \mathrm{x}}$.

É importante ressaltar que os dois tipos de instabilidade não são excludentes: podem ocorrer (e ocorrem) em conjunto ou separadamente. Neste trabalho, os perfis foram escolhidos de tal forma que, 
didaticamente, os dois tipos de instabilidade baroclínica ocorressem separadamente.

\section{PERFIS MÉDIOS VERTICAIS}

Nesta seção, elaboraremos perfis verticais de jatos meridionais e freqüência de empuxo teóricos que satisfaçam as condições para instabilidades apresentadas na seção anterior. Os perfis teóricos escolhidos representam, de forma idealizada, o sistema CB-CCl.

\section{Freqüência de empuxo}

A representação da variação vertical da estratificação é feita através da escolha de um perfil de freqüência de empuxo $(\mathrm{N})$, ajustado exponencialmente, a partir de um perfil médio de $\mathrm{N}$ climatológico da região sudeste do Brasil.

0 perfil ajustado é apresentado na Figura 2, assim como 0 seu gradiente vertical. Sua forma analíitica é dada por

$\mathrm{N}^{2}(\mathrm{z})=\mathrm{c}_{1}+\mathrm{c}_{2} \mathrm{e}^{\left(\mathrm{z} / \mathrm{h}_{1}\right)}+\mathrm{c}_{3} \mathrm{e}^{\left(\mathrm{z} / \mathrm{h}_{2}\right)}$ a)

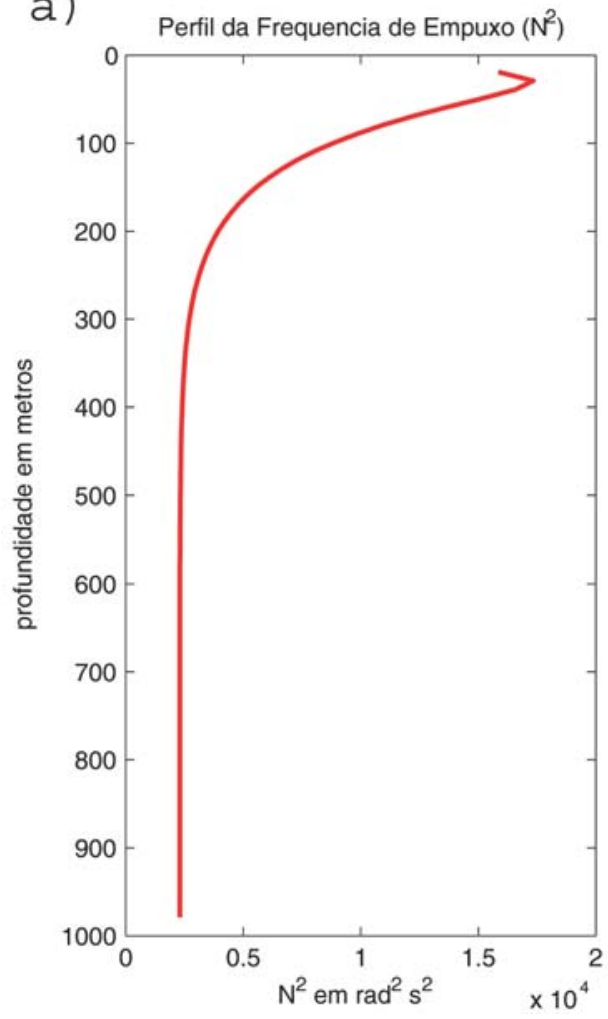

b)

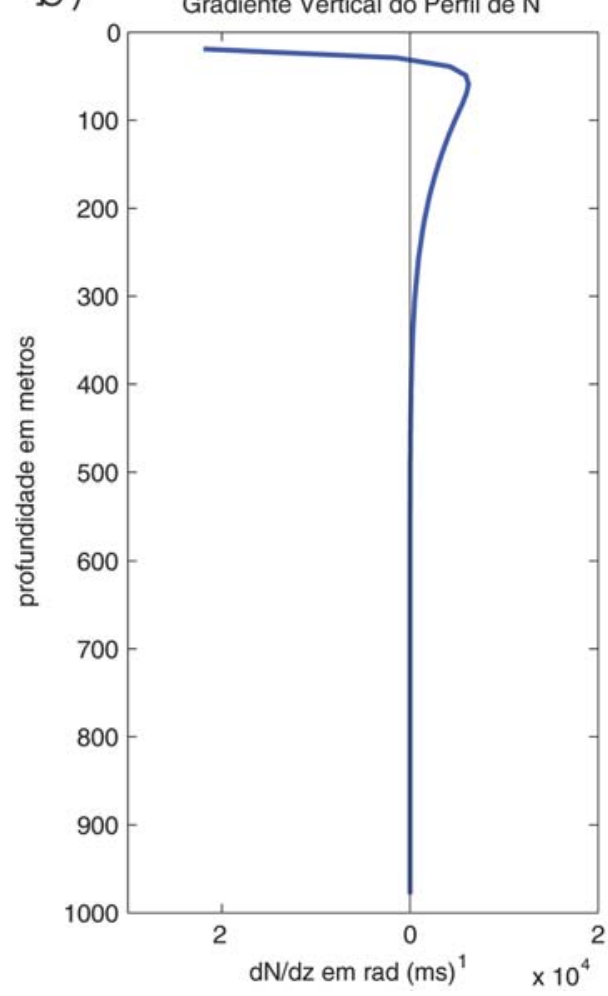

Figura 2 - Perfil do quadrado da frequência de empuxo $\mathrm{N}^{2}$ (Fig. 2a) e o seu gradiente vertical $\frac{\partial \mathrm{N}}{\partial \mathrm{z}}$ (Fig. 2b).

Figure 2 - Profile of the square of the buoyancy frequency $\mathrm{N}^{2}$ (Fig. 2a) and its vertical gradient $\frac{\partial \mathrm{N}}{\partial \mathrm{z}}$ (Fig. 2b). 
onde as constantes $\mathrm{c}_{1^{\prime}} \mathrm{c}_{2^{\prime}} \mathrm{c}_{3^{\prime}} \mathrm{h}_{1}$ e $\mathrm{h}_{2}$ estão listadas na Tabela 1. Esses valores foram escollhidos de forma que o perfil médio da freqüência de empuxo, como dito anteriormente, a um perfil médio de $\mathrm{N}$ climatológico da região sudeste do Brasil.

Tabela 1- Constantes da forma analíica da frequiência de empuxo. Table 1 - Coefficients of the analytical form of the buoyancy frequency.

\begin{tabular}{|c|c|}
\hline$c_{1}$ & $2,31 \times 10^{-05} \mathrm{~s}^{-2}$ \\
\hline$c_{2}$ & $-4,33 \times 10^{-04} \mathrm{~s}^{-2}$ \\
\hline$c_{3}$ & $2,59 \times 10^{-04} \mathrm{~s}^{-2}$ \\
\hline$h_{1}$ & $9,97 \mathrm{~m}$ \\
\hline$h_{2}$ & $72,19 \mathrm{~m}$ \\
\hline
\end{tabular}

\section{Jato Meridional}

Os perfis teóricos de velocidade propostos, bem como seus gradientes verticais, são apresentados na Figura 3 .

Os painéis superiores mostram o perfil de velocidade, denominado "A", assim como o seu gradiente vertical. Analogamente, os painéis

\section{a)}

Perfil Vertical da Velocidade

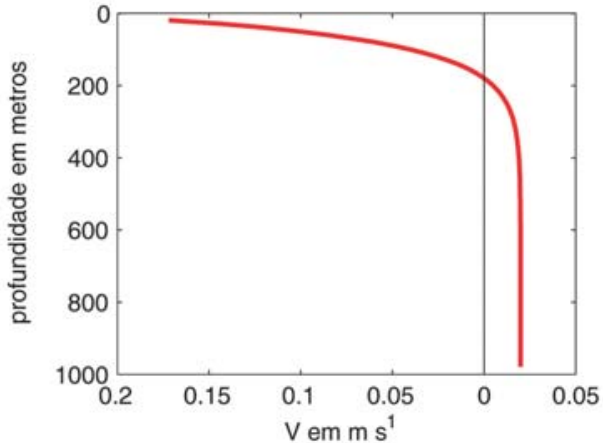

C)

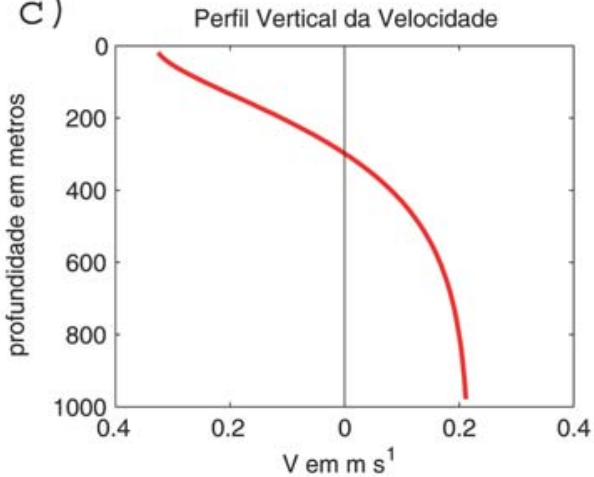

inferiores apresentam o perfil de velocidade, denominado "B", e seu gradiente vertical.

A forma analítica dos perfis segue a dos ajustes de $\mathrm{N}^{2}$ (Equação 36), tratando-se, entretanto, de simulações. Assim,

$$
\overline{\mathrm{v}}=\mathrm{V}_{0}+\mathrm{V}_{1} \mathrm{e}^{(\mathrm{z} / \mathrm{h} 3)}+\mathrm{V}_{2} \mathrm{e}^{(\mathrm{z} / \mathrm{h} 4)},
$$

onde $\mathrm{V}_{0}$ é a velocidade média, $\mathrm{V}_{1} \in \mathrm{V}_{2}$ as amplitudes das velocidades baroclínica, e $\mathrm{h}_{3}$ e $\mathrm{h}_{4}$ as escalas de variação associadas.

A Tabela 2 exibe os valores dessas constantes para os perfis "A" (Figura 3, painel superior) e "B" (Figura 3, painel inferior).

Tabela 2 - Constantes da forma analítica do jatos meridionais "A" e "B". Table 2 - Coefficients of the analytical form of meridional jets "A" and "B".

\begin{tabular}{|c|c|c|}
\hline & Perfil " $A$ " & Perfil " $B$ " \\
\hline$V_{0}$ & $0,02 \mathrm{~ms}^{-1}$ & $0,22 \mathrm{~ms}^{-1}$ \\
\hline$V_{1}$ & $-0,25 \mathrm{~ms}^{-1}$ & $-1,10 \mathrm{~ms}^{-1}$ \\
\hline$V_{2}$ & $-0,10 \mathrm{~ms}^{-1}$ & $0,55 \mathrm{~ms}^{-1}$ \\
\hline$h_{3}$ & $72,19 \mathrm{~m}$ & $200,00 \mathrm{~m}$ \\
\hline$h_{4}$ & $15,00 \mathrm{~m}$ & $100,00 \mathrm{~m}$ \\
\hline
\end{tabular}

b)

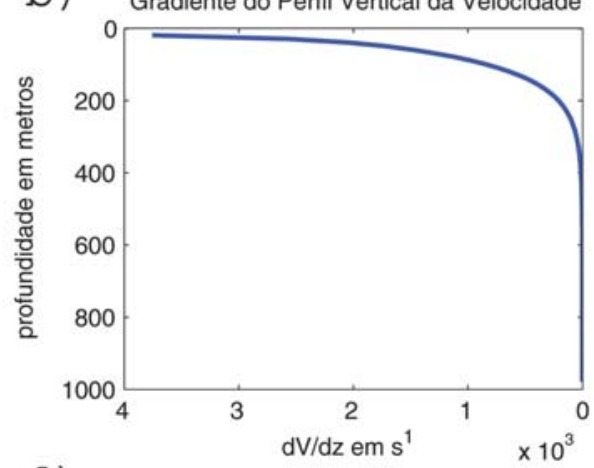

d)

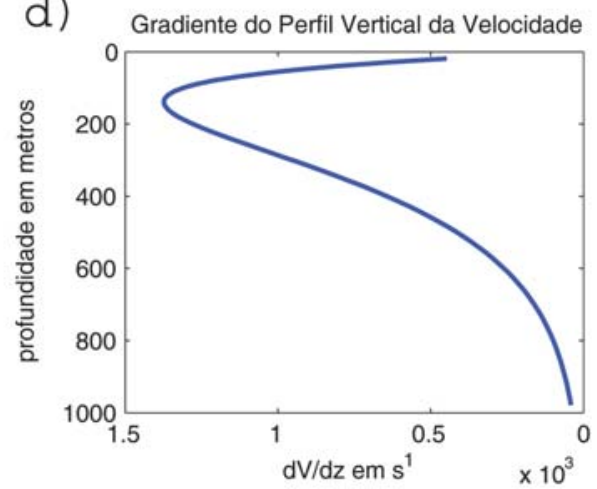

Figura 3 - Perfis verticais de velocidade meridional e 0 correspondente gradiente vertical para os jatos "A" (Fig. 3a e 3b) e "B" (Fig. 3c e 3d). Figure 3 - Vertical profiles of meridional velocity and the corresponding vertical gradients for jets "A" (Fig. 3a and 3b) and "B" (Fig. 3c and 3d). 
a)

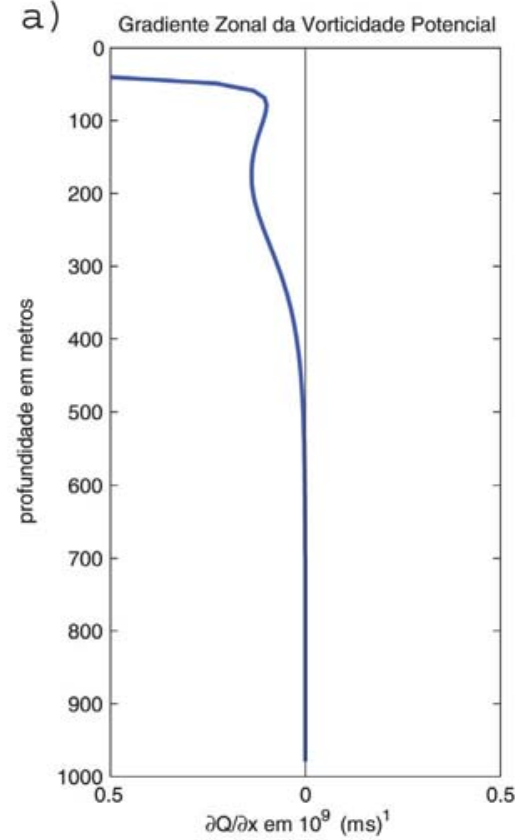

b)

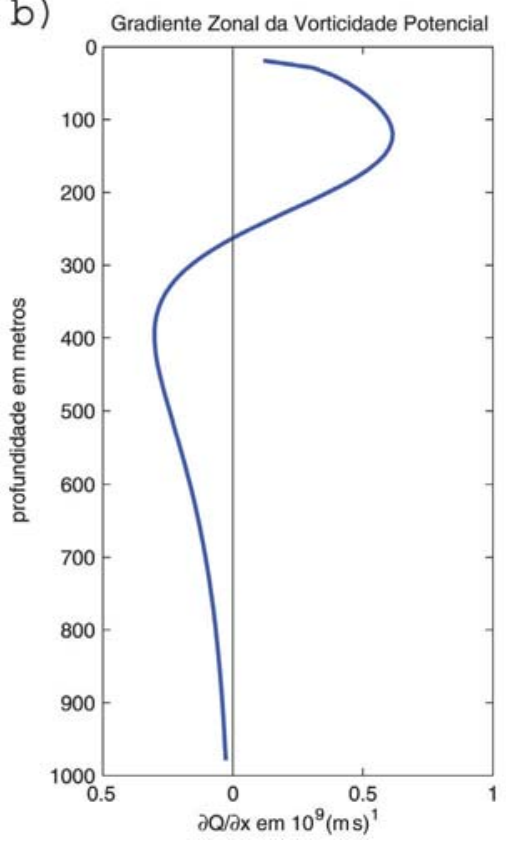

Figura 4 - Perfil vertical do gradiente zonal de vorticidade potencial para 0 jato "A" (Fig. 4a) e 0 jato "B" (Fig. 4b).

Figure 4 - Vertical profile of the zonal gradient of potencial vorticity for "A" (Fig. 4a) and jet "B" (Fig. 4b).

\section{Gradiente Zonal de Vorticidade Potencial}

A Figura 4 exibe os perfis médios do gradiente zonal de vorticidade potencial, calculados a partir da Equação (21), para os perfis "A" e "B". Nota-se que, de acordo com as condições necessárias de instabilidade baroclínica, ambos os perfis satisfazem as condições necessárias para sua ocorrência. Verifica-se que, satisfazendo-se a primeira condição de instabilidade, automaticamente as outras duas condições serõo satisfeitas.

Entretanto, as escolhas das formas de para os perfis de " $\mathrm{A}$ " $\mathrm{e}$ "B" mostram-se evidentes. 0 perfil de $\frac{\partial \overline{\mathrm{q}}}{\partial \mathrm{x}}$ do jato "A" apresenta alta variação próximo à superfície. Ou seja, 0 jato " $\mathrm{A}$ " é um candidato natural a apresentar instabilidade de borda. Já, o perfil de jato " $\mathrm{B}$ ", apresenta troca de sinal em $265 \mathrm{~m}$ de profundidade, distante de ambos os limites verticais. 0 valor pequeno de $\frac{\partial \overline{\mathrm{q}}}{\partial \mathrm{x}}$ nestes limites sugere que 0 jato "B" seja um candidato natural à instabilidade baroclínica interna.
De volta a Equação (35), pode-se evidenciar as suposições anteriores com argumentos mais quantitativos. Considerando-se que os três termos da Equação (35) devem ter aproximadamente a mesma ordem, 0 nível de acoplamento precisa estar localizado numa região próxima à mudança de sinal de $\frac{\partial \bar{q}}{\partial \mathrm{x}}$. Isto porque a única forma de que os incrementos da integral do terceiro membro da Equação (35) tenham aproximadamente a mesma contribuição que toda a integral é que, na região onde os valores de $|\bar{v}-c| \rightarrow 0,0$ termo integral apresente valores de $\frac{\partial \overline{\mathrm{q}}}{\partial \mathrm{x}}$ com sinais opostos próximos ao nível de acoplamento.

\section{Cálculo de Instabilidade}

Os parâmetros do modelo unidimensional composto pelas Equações (26), (27) e (28) foram fixados para os dois perfis: a largura do canal foi escolhida $\mathrm{L}=100 \mathrm{~km}$ e as taxas entre a inclinação do fundo em relação à inclinação das isopicnais profundas foram 
a) Velocidade de Fase

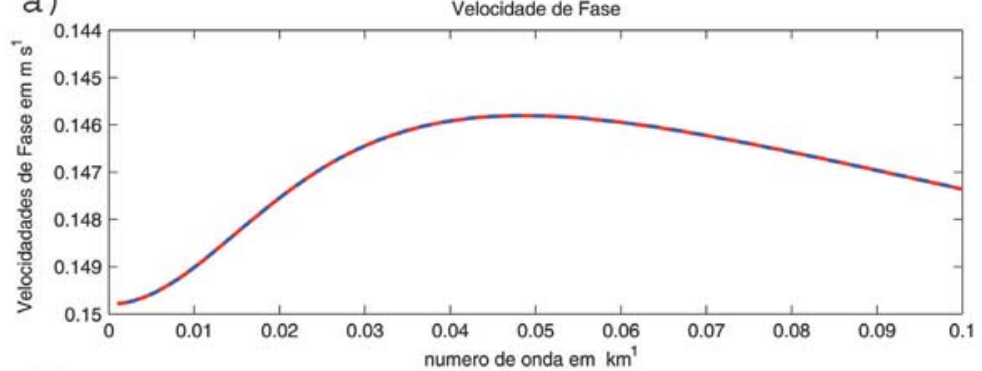

b)

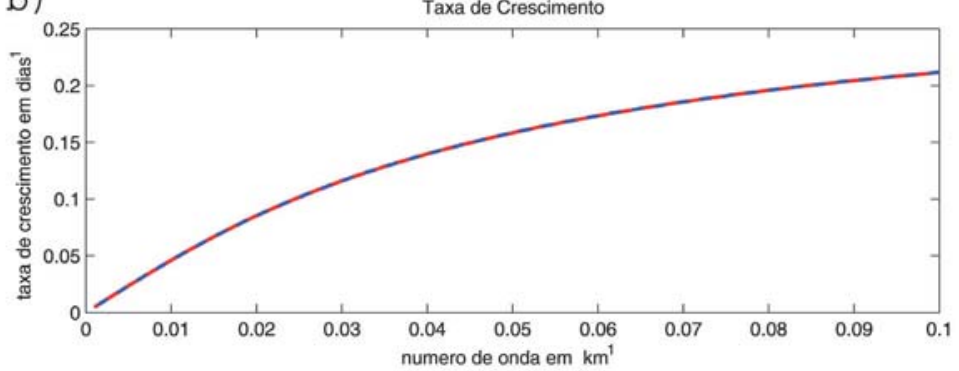

Figura 5 - Velocidade de fase das ondas instáveis (Fig. 5a) e a taxa de crescimento da ondas instáveis (Fig. 5b) do jato " $A$ ".

Não há diferença observável entre os casos $\frac{\partial \mathrm{h}^{*}}{\partial \mathrm{x}}=0 \mathrm{e} \frac{\partial \mathrm{h}^{*}}{\partial \mathrm{x}}=10$.

Figure 5 - Phase velocity of the unstable waves (Fig. 5a) and the growth rate of unstable waves (Fig. 5b) of jet "A".

There is no notable difference between $\frac{\partial \mathrm{h}^{*}}{\partial \mathrm{x}}=0$ and $\frac{\partial \mathrm{h}^{*}}{\partial \mathrm{x}}=10$.

a)

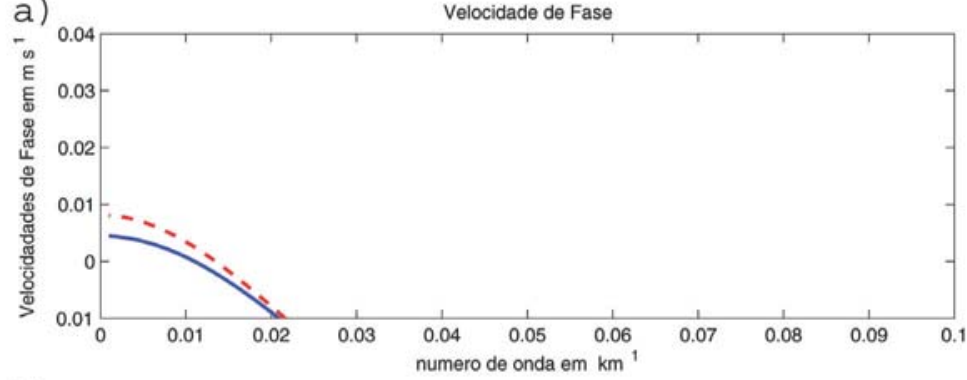

b)

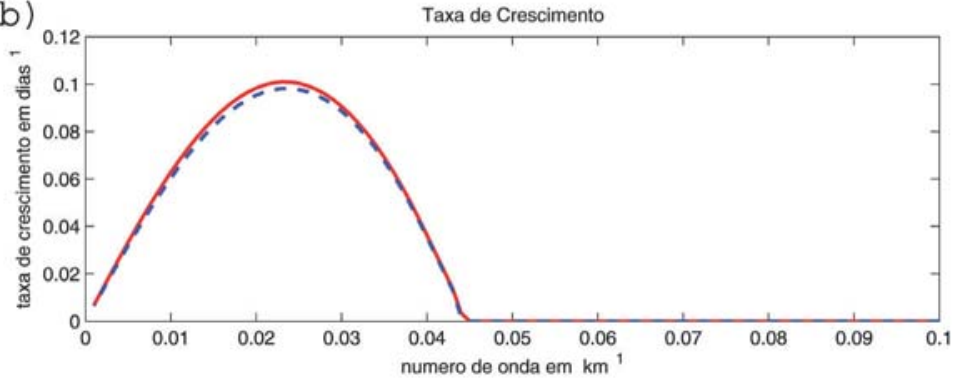

Figura 6 - Velocidade de fase das ondas instáveis (Fig. 6a) e a taxa de crescimento da ondas instáveis (Fig. 6b) do jato "B".

Curvas sólidas correspondem ao caso para 0 qual $\frac{\partial \mathrm{h}^{*}}{\partial \mathrm{x}}=0$, e curvas tracejadas correspondem ao de $\frac{\partial \mathrm{h}^{*}}{\partial \mathrm{x}}=10$.

Figure 6 - Phase velocity of the unstable waves (Fig. 6a) and the growth rate of unstable waves (Fig. 6b) of jet "B". The solid lines correspond to the case where $\frac{\partial \mathrm{h}^{*}}{\partial \mathrm{x}}=0$, and the dashed lines correspond to the case $\frac{\partial \mathrm{h}^{*}}{\partial \mathrm{x}}=10$. 
consideradas $\frac{\partial \mathrm{h}^{*}}{\partial \mathrm{x}}=0$ (fundo plano) e $\frac{\partial \mathrm{h}^{*}}{\partial \mathrm{x}}=10$ (a fim de simular 0 talude continental de forma rudimentar).

Os resultados do cálculo das propriedades lineares de instabilidade são mostrados nas Figuras 5 (para 0 jato " $\mathrm{A}$ ") e 6 (para o jato "B"), onde as curvas sólidas representam a solução para configurações de oceano com fundo plano e as curvas tracejadas para configurações considerando presença de inclinação de fundo. A Figura 7 exibe a estrutura vertical da onda mais instável para os jatos "A" (Figuras 7a e 7b) e "B" (Figuras 7c e 7d).

Inicia-se a apresentação e discussão dos resultados obtidos envolvendo configurações de um oceano com o fundo plano. Para 0 perfil "A", a região onde localiza-se o nível de acoplamento é em
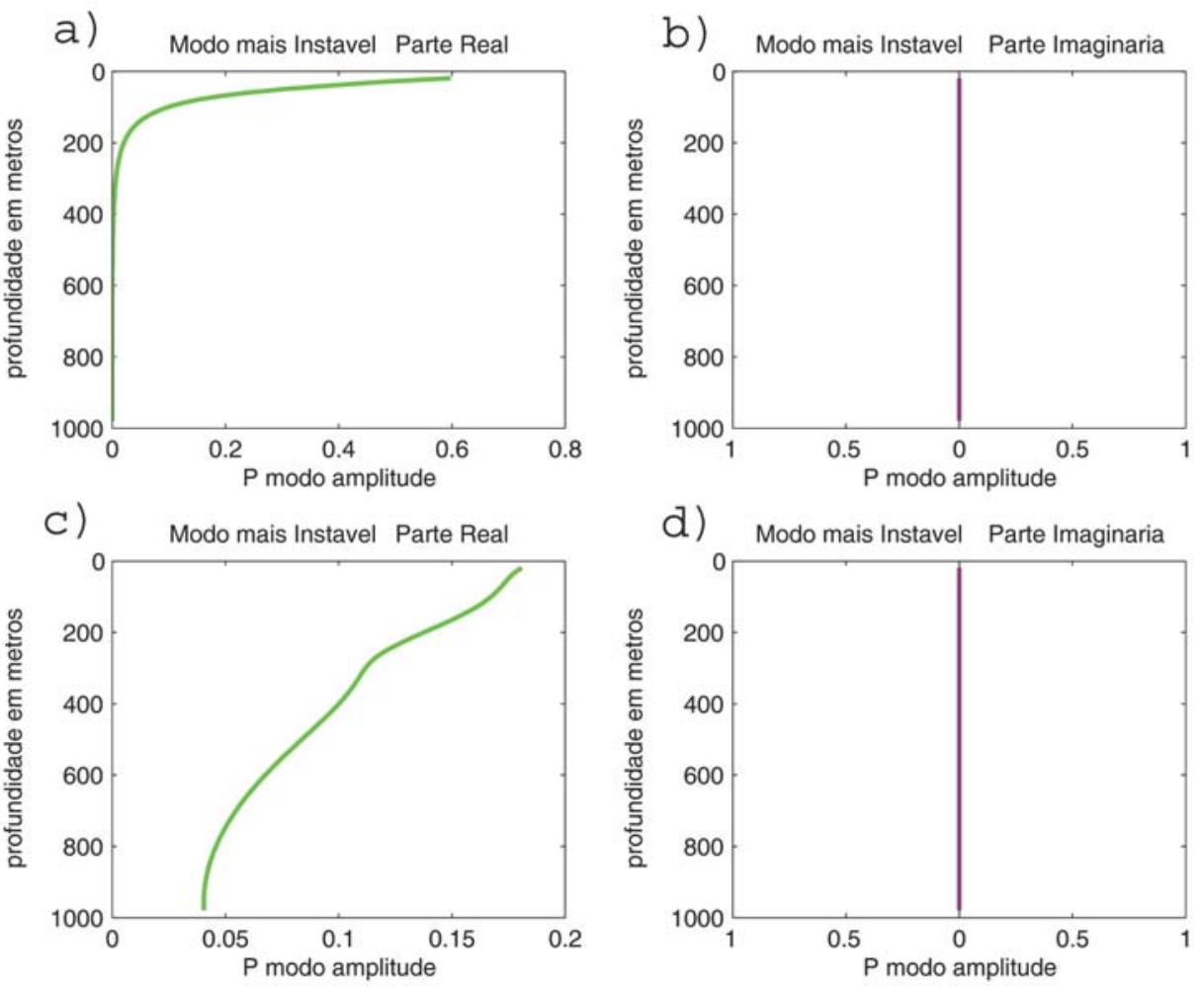

Figura 7 - Perfil vertical da amplitude do modo mais instável dos jatos “A” (Fig. 7a e 7b) e "B" (Fig. 7c e 7d). Figure 7 - Vertical profile of the amplitude in the most unstable wave of jet "A" (Fig. Ta and 7b) and jet "B" (Fig. Tc and 7d).

torno de $30 \mathrm{~m}$. Observamos, no perfil de velocidade da Figura $3 a$, que a esta profundidade existem valores de velocidade da ordem $-0,15 \mathrm{~ms}^{-1}$. Estes correspondem a velocidade de fase média das ondas instáveis apresentadas no diagrama de dispersão da Figura 5b. Neste diagrama, observamos que as ondas são apenas ligeiramente dispersivas e que se aproximam assintoticamente do valor de em superfície. Tal comportamento é típico de instabilidade de borda. Ondas mais curtas apresentam maiores taxas de crescimento num regime instável, onde apenas ondas infinitamente longas não crescem em amplitude.

A Figura 7a ilustra a estrutura vertical de $\Psi(\mathrm{z})$ para a onda mais instável exibida na Figura 5b. Esta estrutura mostra um rápido decréscimo vertical e apenas valores de amplitude razoáveis nos primeiros
100 m. Trata-se, portanto, de uma onda instável tipo Eady, que confirma um regime em que o fenômeno da instabilidade baroclínica de borda está isolado.

Para o perfil de "B", de acordo com a Figura 3a, a região onde $\frac{\partial \bar{q}}{\partial x}$ muda de sinal é localizada aproximadamente em $265 \mathrm{~m}$ de profundidade. Pela análise das Figuras $6 a *$ e $6 b$, onde temos a taxa de crescimento das ondas instáveis para $\frac{\partial \mathrm{h}^{*}}{\partial \mathrm{x}}=10$, fica evidente que os valores de velocidade onde há crescimento das ondas variam entre 0,04 e $0,01 \mathrm{~cm} \mathrm{~s}^{-1}$. Verificando no perfil de velocidade, nota-se que a região de acoplamento está compreendida entre 240 e 360m de profundidade. Este nível de acoplamento encontra-se afastado dos contornos, caracterizando existência de instabilidade interna. Em oposição 
aos resultados obtidos para 0 jato "A", o regime de ondas instáveis obtido é limitado para ondas de comprimento relativamente longos. A Figura 7c exibe a estrutura vertical de $\Psi(\mathrm{z})$ para a onda mais instável exibida na Figura 6b. Essa onda apresenta amplitude considerável ao longo de toda a coluna de água, com valor junto ao fundo representando 1/4 do valor de superfície. Tal configuração é também característica de instabilidade baroclínica interna, onde variações na vertical de gradientes horizontais de densidade facilitam a drenagem de energia pelas perturbações.

Considerem-se, agora, os resultados obtidos quando utilizam-se configurações de oceano com um fundo inclinado, isto é, um experimento onde valores de $\frac{\partial \mathrm{h}^{*}}{\partial \mathrm{x}}=10$ foram utilizados. Verifica-se nas Figuras $5 \mathrm{a}$ e $5 b$ que, para o perfil " $A$ " não há mudança de comportamento em relação ao valor de $\frac{\partial \mathrm{h}^{*}}{\partial \mathrm{x}}=0$. As curvas tracejadas e sólidas se superpõem. Isto é, obviamente, conseqüência de que já que obtivemos ondas fortemente aprisionadas junto à superfície, o efeito do fundo não é sentido por essas. Logo, pela Equação (35), o balanço principal dos termos para ocorrência de instabilidade de borda junto à superfície se dá entre 0 primeiro e terceiro termos da equação.

Já para 0 perfil "B", Figuras 6a e $6 \mathrm{~b}$, quando $\frac{\partial \mathrm{h}^{*}}{\partial \mathrm{x}}=10$, as velocidades das ondas sofrem um desvio, principalmente na região de velocidades negativas, e a taxa de crescimento é ligeiramente menor, em relação a $\frac{\partial \mathrm{h}^{*}}{\partial \mathrm{x}}=0$. Em outras palavras, 0 efeito da inclinação do fundo na mesma direção do talude continental é estabilizador. Pela Equação (35), o segundo termo adquire importância relativa em contraponto ao primeiro termo (referente à condição de contorno de superfície). A expressão entre colchetes se anula devido à dominância e inversão de sinal nos incrementos da integral do terceiro termo, enquanto o segundo termo exerce mero efeito atenuador no crescimento das amplitudes.

\section{Considerações Finais}

0 modelo de Johns (1988) representa um exemplo simples da instabilidade de cisal hamento vertical em escoamentos fluidos geofísicos. Neste trabalho, apresentamos a variação desse modelo para jatos meridionais no plano $\mathrm{f}$, utilizando uma estratificação simulada da região sudeste brasileira e perfis teóricos idealizados, motivados pelo cisalhamento vertical do sistema CB-CCl. 0 principal objetivo foi representar dois tipos de instabilidade baroclínica que ocorrem comumente no oceano: instabilidade de borda e instabilidade interna. Desta forma, pode-se obter o comportamento das ondas instáveis, para os padrões de perfis verticais apresentados, através do cálculo das taxas de crescimento e da inclinação do fundo oceânico, para os casos de instabilidade de borda e instabilidade interna.

Para 0 perfil de velocidade " $\mathrm{A}$ ", obteve-se, isoladamente, a instabilidade de borda, com a velocidade de fase e taxa de crescimento das ondas instáveis mostradas nas Figuras $5 a$ e $5 b$. Tal instabilidade é confirmada pela presença de uma onda instável do tipo Eady, na amplitude vertical da função de onda perturbada $\Psi$ ' (Figura 7a). Analisando-se o perfil de velocidades "B" obtivemos, ao contrário do caso " $\mathrm{A}$ ", instabilidade interna, que tem a velocidade de fase e taxa de crescimento das ondas instáveis mostradas nas Figuras 6a e 6b. Essa instabilidade é também obtida isoladamente e pode-se confirmá-la, também, pelo exame da amplitude vertical da função de onda perturbada $\Psi$ ', que apresenta como onda mais instável uma onda de grande amplitude, 0 que é característico da instabilidade interna (Figura 7c).

Os resultados obtidos também mostram que, no caso de instabilidade de borda, ondas mais curtas são mais instáveis e suas velocidades de fase aproximam-se da velocidade do jato na borda superior. Para o caso de instabilidade interna, as ondas instáveis ocorrem em um regime de comprimentos de onda relativamente longos, com a velocidade de fase dessas ondas apresentando valores próximos aos valores de velocidade do jato na região de inversão de fluxo. Com relação aos efeitos de inclinação do fundo, as ondas geradas na instabilidade de borda apresentam-se aprisionadas na superfície, não havendo, portanto, modificação alguma com a inclinação do fundo, enquanto as ondas geradas por instabilidade interna sofrem um decréscimo na sua taxa de crescimento e um aumento na velocidade de fase das ondas mais longas para uma inclinação positiva de $\mathrm{h}^{*}$, mostrando 0 efeito estabilizador da inclinação do fundo nas ondas geradas por instabilidade interna.

No oceano, instabilidades baroclínicas e barotrópica (devido a cisalhamento horizontal) ocorrem simultaneamente. Geralmente, em sistemas de corrente de contorno oeste, a instabilidade baroclínica interna é o mecanismo inicial e desencadeador do processo de formação de meandros de amplitude finito e vórtices isolados, fundamental para a troca de propriedades físicas, químicas e biológicas entre as diferentes regiões oceânicas e a atmosfera.

\section{Agradecimentos}

Este trabalho faz parte do Projeto DINBRAS, financiado pela FAPESP (98/00572-2), a bolsa de produtividade em pesquisa do CNPq (3005821/96-0 - RN), do primeiro autor, e, a bolsa de doutorado FAPESP (02/13006-2), do terceiro autor. 


\section{REFERÊNCIAS}

CALADO, L. Dinâmica da formação dos meandros e vórtices da Corrente do Brasil ao largo do Sudeste Brasileiro. 2001. Dissertação (Mestrado)Instituto Oceanográfico da Universidade de São Paulo, São Paulo, 2001. CHARNEY, J. G. The dynamics of long waves in a baroclinic westerly current. J. Meteorol., [S.I.], v. 4, p. 135-162, 1947.

; STERN, M. On the stability of internal baroclinic jets in a rotating atmosphere. J. Atmos. Sci., [S.I.], v. 19, p. 159-172, 1962. CUSHMAN-ROISIN, B. Introduction to Geophysical Fluid Dynamics. [S.I.]: Prentice Hall, 1994.

EADY, E. J. Long waves and cyclone waves. Tel/us, [S.I.], v. 1, p. 33-52, 1949.

EVANS, D. L.; SIGNORINI, S. R. Vertical structure of the Brazil current. Nature, [S.I.], n. 315, p. 48-50, 1985.

FERNANDES, A. M. Dinâmica baroclínica da Corrente do Brasil na região sudeste. Dissertação (Mestrado)- Instituto Oceanográfico da Universidade de São Paulo, São Paulo, 2001.
FLLERL, G. R. Models of vertical structure and the calibration of two-layer models. Dyn. Atmos. Oceans, [S.I.], v. 2, p. 341-381, 1978.

GREEN, A. E. A problem in baroclinic stability. Quart. J. Roy. Metorol. Soc., [S.I.], v. 86, p. 237-251, 1960

GILL, A. E.; GREEN J. S. A.; SIMMONS A. J. Energy partition in the largeescale ocean circulation and the productions of mid-ocean eddies. DeepSea Res., [S.I.], v. 21, p. 499-528, 1974.

JOHNS, W. E. One-dimensional baroclinically unstable waves on the Gulf Stream potential vorticity gradient near Cape Hatteras. Dyn. Atm. Ant, Oc., [S.I.], v. 11, p. 323-350, 1988.

PEDLOSKY, J. Geophysical fluid dynamics. New York: Springer-Verlag, 1979.

SILVEIRA, I. C. A. da et al. A Corrente do Brasil ao largo da Costa este Brasileira. Revista Brasileira de Oceanografia, [S.I.], v. 48, n. 2, p. 171183 (C11), 2001.

\section{NOTAS SOBRE OS AUTORES}

Ilson Carlos Almeida da Silveira é Doutor do Departamento de Oceanografia Física do Instituto Oceanográfico da Universidade de São Paulo (IOUSP), onde leciona e desenvolve pesquisas desde 1997. Orientador de cinco doutorandos, dois mestrandos e dois alunos de iniciação científica, além de ter formado um doutor e quatro mestres. Graduado em Oceanografia pela Universidade do Estado do Rio de Janeiro (UERJ), em 1984. Mestre em Oceanografia Física pelo IOUSP, em 1990 e Doutor pela University of New Hampshire em 1996, fez seu pós-doutoramento em Rhode Island no Graduate School of Oceanography (GSO), entre 1996 e 1997. Desde então participou de vários projetos de pesquisa na qualidade de responsável e colaborador. Sua linha de pesquisa compreende a circulação de meso e larga escalas do oceano, voltada para teoria da instabilidade hidrodinâmica e para 0 estudo da dinâmica das correntes de contorno oeste, principalmente da Corrente do Brasil, através do desenvolvimento e utilização de modelos analíticos, semi-analíticos e numéricos.

Leandro Calado é aluno do programa de Doutorado em Oceanografia Física do Instituto Oceanográfico da Universidade de São Paulo (IOUSP). Graduado em Licenciatura em Física na Universidade Federal do Rio de Janeiro (UFRJ) e Mestre em Oceanografia Física pelo IOUSP. Sua linha de pesquisa compreende o estudo da circulação de meso e larga escalas do oceano, principalmente através de utilização de modelos numéricos, abordando os processos envolvidos na dinâmica da Corrente do Brasil.

Cayo Prado Fernandes Francisco é aluno do programa de Doutorado em Oceanografia Física do Instituto Oceanográfico da Universidade de São Paulo (USP). Bacharel em Física em 2001 pelo IFUSP. Suas áreas de interesse compreendem a dinâmica de fluidos geofíisicos, instabilidades lineares e não lineares em fluidos e supercondutividade e superfluidez teóricas. 